九州大学学術情報リポジトリ

Kyushu University Institutional Repository

\title{
TENEBRIONIDAE OF THE NANSEI ISLANDS II (COLEOPTERA)
}

Chujo, Michitaka

https://doi.org/10.5109/2367

出版情報: ESAKIA. 10，pp.1-19，1977-02-15. Hikosan biological laboratory，Faculty of Agriculture, Kyushu University バージョン :

権利関係 : 


\title{
TENEBRIONIDAE OF THE NANSEI ISLANDS II (COLEOPTERA)*
}

\author{
Michitaka Chû Jô \\ Hikosan Biological Laboratory, Faculty of Agriculture, \\ Kyushu University, Hikosan, Fukuoka 824-07, Japan
}

\begin{abstract}
Synopsis: Nine species of the Tenebrionidae are newly recorded from Nakanoshima I., 3 from Akusekijima I., 1 from Takarajima I., 1 from Kikaijima I., 4 from Amami-Oshima I., 7 from Tokunoshima I. and 6 from Okinoerabujima I. Three new taxa are Tetraphyllus lunuliger Zaevis from Amami-Oshima I., Tokunoshima I. and Okinawa I., Phaedis (Phaedis) helopioides amamianus from Amami-Oshima I. and Plesiophthalmus makiharai from Tokunoshima I.
\end{abstract}

\section{NAKANOSHIMA I.}

\section{Opatrini}

1. Gonocephalum okinawanum M. T. Chûjô, 1963

Spec. exam.: None (recorded by Z. Kaszab, 1964).

Bolitophagini

†2. Byrsax niponicus Lewis, 1894

Spec. exam.: 1 ex., Nakanoshima I., Nansei Is., Japan, 2. vii. 1973, H. Irie leg.

\section{Diaperini}

3. Diaperis sanguinipennis sinensis Gebien, 1925

* Contribution from the Hikosan Biological Laboratory, Faculty of Agriculture, Kyushu University, Hikosan (Ser. 2, No. 51).

$\dagger$ The dagger shows that the species has hitherto been unrecorded from the island. 
Spec. exam.: 3 exs., Nakanoshima, Is. Tokara, 7. vii. 1960, M. Sato leg.; 3 exs., id., 9. vii. 1960, M. Sato leg.; 1 ex., Nakanoshima, Toshima vill., Kagoshima pref., 24. vi. 1973, H. Makihara leg. ; 2 exs., Nakanoshima, Nansei Is., Japan, 2. vii. 1973, H. Irie leg.

4. Platydema marseuli Lewis, 1894

Spec. exam.: 1 ex., Nakanoshima, Tokara Is., 13. vii. 1960, Y. Hama leg.; 2 exs., Nakanoshima, Nansei Is., Japan, 27. vi. 1973, H. Irie leg. ; 1 ex., id., 2. vii. 1973, H. Irie leg.

5. Platydema pallidicolle (Lewis, 1894)

Spec. exam.: 2 exs., Nakanoshima Is., Tokara, 5. vii. 1960, M. Sato leg.

†6. Platydema recticorne Lewis, 1894

Spec. exam.: 3 exs., Nakanoshima, Is. Tokara, 5. vii. 1960, M. Sato leg.

7. Platydema subfascia subfascia (Walker, 1858)

Spec. exam.: 19 exs., Nakanoshima, Is. Tokara, 5. vii. 1960, M. Sato leg.; 10 exs., id., 7. vii. 1960, M. Sato leg.; 2 exs., Nakanoshima, Toshima vill., Kagoshima pref., 24. vi. 1973, H. Makihara leg.; 2 exs., Nakanoshima, Nansei Is., Japan, 1. vii. 1973, H. Irie leg. ; 1 ex., id., 26. iv. 1975, H. Irie leg. ; 3 exs., id., 1. v. 1975, H. Irie leg.

†8. Basanus amamianus M. T. Chûjô, 1966

Spec. exam.: 6 exs., Is. Nakanoshima, Tokara, 24. vii. 1969, M. Sakai leg.; 1 ex., Nakanoshima, Nansei Is., Japan, 2. vii. 1973, H. Irie leg. 9. Ceropria induta induta (Wiedemann, 1819)

Spec. exam. : 1 ex., Nakanoshima, Is. Tokara, 5. vii. 1960, M. Sato leg.; 1 ex., id., 7. vii. 1960, M. Sato leg. ; 1 ex., Nakanoshima Is., Tokara-Islands, 28. vii. 1964, S. Ohga leg.; 2 exs., id., 28. vii. 1964, S. Ohga leg. ; 1 ex., Nakanoshima, Toshima vill., Kagoshima pref., 24. vi. 1973, H. Makihara leg. ; 4 exs., id., 25. vi. 1973, H. Makihara leg.; 2 exs.(at light), id., 25. vi. 1973, H. Makihara leg.; 2 exs., Nakanoshima, Nansei Is., Japan, 1. v. 1975, H. Irie leg.; 4 exs., Nakanoshima, Kagoshima, Japan, 19-20. vii. 1975, H. Makihara leg.; 1 ex., id., 9-10. viii. 1975, H. Makihara leg.

†10. Ceropria laticollis Fairmaire, 1903

Spec. exam.: 1 ex., Nakanoshima, Toshima vill., Kagoshima pref., 5. iv. 1974, H. Makihara leg.

11. Pentaphyllus philippinensis Kaszab, 1956

Spec. exam.: 4 exs., Nakanoshima, Is. Tokara, 5. vii. 1960, M. Sato leg.; 1 ex., id., 11. vii. 1960, M. Sato leg.; 7 exs., Nakanoshima, Nansei Is., Japan, 4. iv. 1974, H. Irie leg. 


\section{Phrenapatini}

\section{Tagalus tokaranus Nakane, 1963}

Spec. exam.: 1 ex., Nakanoshima, Tokara Is., Japan, 18. v. 1964, H. Shima leg.

\section{Ulomini}

†13. Tribolium (Tribolium) castaneum (Herbst, 1797)

Spec. exam.: 12 exs., Nakanoshima, Nansei Is., Japan, 27. vi. 1973, H. Irie leg.

14. Uloma excisa excisa Gebien, 1913

Spec. exam. : 1 ex., Nakanoshima, Is. Tokara, 5. vii. 1960, M. Sato leg. ; 1 ex., id., 13. vii. 1960, M. Sato leg. ; 12 exs., Is. Nakanoshima, Tokara Islands, 24. v. 1962, M. Sato leg. ; 1 ex., id., 24. vii. 1964, A. Tanaka leg. ; 1 ex., Sokonashinuma, Tokara Is., Japan, 24. vii. 1964, H. Shima leg.; 3 exs., Nakanoshima, Nansei Is., Japan, 1. vii. 1973, H. Irie leg.; 7 exs., id., 2. vii. 1973, H. Irie leg. ; 3 exs., id., 27. iv. 1975, H. Irie leg.

15. Alphitobius laevigatus (Fabricius, 1781)

Spec. exam.: None (recorded by Z. Kaszab, 1964).

16. E utochia lateralis (Boheman, 1858)

Spec. exam.: None (recorded by T. Nakane, 1963).

17. H ypophloeus (Paraphloeus) gentilis (Lewis, 1894)

Spec. exam. : 1 ex., Nakanoshima, Nansei Is., Japan, 2. vii. 1973, H. Irie leg.; 2 exs., id., 30. iv. 1975, H. Irie leg.

\section{Tenebrionini}

18. M enephilus arciscelis Marseul, 1876

Spec. exam. : 1 ex., Nakanoshima, Nansei Is., Japan, 1. vii. 1973, H. Irie leg.; 1 ex., id., 27. iv. 1975, H. Irie leg.

19. Cryphaeus punctulatus (Lewis, 1894)

Spec. exam.: None (recorded by T. Nakane, 1963).

20. Cryphaeus satoi Kaszab, 1964

Spec. exam.: 1 ex.(holotype), Nakanoshima, Tokara Is., 13. vii. 1960, Y. Hama leg.; 1 ex., Nakanoshima, Toshima vill., Kagoshima pref., 24. vi. 1973, H. Makihara leg. ; 2 exs., Nakanoshima, Nansei Is., Japan, 1. vii. 1973, H. Irie leg.; 1 ex., id., 2. vii. 1973, H. Irie leg. ; 3 exs., id., 27. iv. 1975, H. Irie leg. ; 2 exs., id., 29. iv. 1975, H. Irie leg.; 1 ex., Nakanoshima, Kagoshima, Japan, 19-20. vii. 1975, H. Makihara leg. 


\section{Cnodalonini}

†21 Hemicera alternata nodokai Nakane, 1963

Spec. exam.: 2 exs., Nakanoshima Is., Tokara-Islands, 28. vii. 1964, S. Ohga leg.; 1 ex., Nakanoshima, Nansei Is., Japan, 1. vii. 1973, H. Irie leg. ; 1 ex., id., 5. iv. 1974, H. Irie leg. ;1 ex., id., 27. iv. 1975, H. Irie leg.; 3 exs., id., 1. v. 1975, H. Irie leg. ; 1 ex., Nakanoshima, Kagoshima, Japan, 19-20. vii. 1975, H. Makihara leg.

†22. Phaedis (Phaedis) helopioides purpurascens Nakane, 1968

Spec. exam.: 2 exs., Nakanoshima, Nansei Is., Japan, 2. vii. 1973, H. Irie leg.; 9 exs., id., 4. vii. 1973, H. Irie leg. ; 1 ex., id., 6. iv. 1974, H. Irie leg. ; 3 exs., id., 1. v. 1975, H. Irie leg. ; 1 ex., id., 2. v. 1975, H. Irie leg.; 2 exs., Nakanoshima, Kagoshima, Japan, 19-20. vii. 1975, H. Makihara leg.; 1 ex., id., 9-10. viii. 1975, H. Makihara leg.

23. O briomaia palpalis palpaloides Nakane, 1963

Spec. exam.: 4 exs., Nakanoshima, Toshima vill., Kagoshima pref., 24. vi. 1973, H. Makihara leg. ; 2 exs., id. 26. vi. 1973, H. Makihara leg. ; 2 exs., Nakanoshima, Nansei Is., Japan, 1. vii. 1973, H. Irie leg. ; 1 ex., id., 2. vii. 1973, H. Irie leg. ; 1 ex., Nakanoshima, Kagoshima, Japan, 19-20. vii. 1975, H. Makihara leg.

†24. O briomaia semiviolacea Nakane, 1968

Spec. exam. : 1 ex., Nakanoshima, Nansei Is., Japan, 1. vii. 1973, H. Irie leg.; 3 exs., id., 2. vii. 1973, H. Irie leg.; 1 ex., id., 27. iv. 1975, H. Irie leg.; 1 ex., Nakanoshima, Kagoshima, Japan, 19-20. vii, 1975, H. Makihara leg.

\section{Misolampini}

25. Paramisolampidius tokarensis (Nakane, 1963)

Spec. exam. : 1 ex.(holotype), Nakanoshima, Is. Tokara, 13. vii. 1960, M. Sato leg. ;1 ex., Nakanoshima, Nansei Is., Japan, 2. vii. 1973, H. Irie leg. ; 1 ex., id., 28. iv. 1975, H. Irie leg.

\section{Helopini}

26. Tarpela brunnea konoi Nakane, 1963

Spec. exam. : 2 exs., Nakanoshima, Nansei Is., Japan, 6. iv. 1974, H. Irie leg. ; 2 exs., id., 28. iv. 1975, H. Irie leg.; 1 ex., id., 30. iv. 1975, H. Irie leg.

\section{Strongyliini}

27. Strongylium cultellatum Mäklin, 1866

Spec. exam. : None (recorded by Z. Kaszab, 1964). 
†28. Strongylium marseuli marseuli Lewis, 1894

Spec. exam.: 1 ex., Nakanoshima, Toshima vill., Kagoshima pref., 24. vi. 1973, H. Makihara leg. ; 1 ex., Nakanoshima, Nansei Is., Japan, 27. vi. 1973, H. Irie leg. ; 2 exs., id., 30. vi. 1973, H. Irie leg.

AKUSEKI JIMA I.

\section{Diaperini}

†1. Diaperis sanguinipennis sinensis Gebien, 1925

Spec. exam. : 1 ex., Akuseki-jima, Tokara Is., 30. vii. 1969, M. Sato leg.

†2. Ceropria induta induta (Wiedemann, 1819)

Spec. exam. : 1 ex., Akuseki-jima, Tokara Is., 19. v. 1962, M. Sato leg.

\section{Misolampini}

†3. Paramisolampidius tok arensis (Nakane, 1963)

Spec. exam.: 1 ex., Akuseki-jima, Tokara Is., 17. v. 1962, M. Sato leg.

TAKARA JIMA I.

\section{Opatrini}

1. Gonocephalum moluccanum Blanchard, 1853

Spec. exam. : 1 ex., Takarajima, Is. Tokara, 2. vii. 1960, M. Sato leg.; 2 ex s., id., 2. vii. 1960, Y. Hama leg.; 1 ex., id., 3. vii. 1960, M. Sato leg.; 2 exs., Takarajima, Tokara Is., Japan, 13. vii. 1964, S. Oga leg. ; 2 ex s., id., 18. vii. 1964, H. Shima leg. ; 1 ex., TakarashimaIs., Tokara-Islands, 20. vii. 1964, S. Ohga leg. ; 2 exs., Oike, Takarajima, Tokara Is., Japan, 21. vii. 1964, S. Oga leg.

2. Gonocephalum okinawanum M. T. Chûjô, 1963

Spec. exam.: 1 ex.(holotype), Hentona, Okinawa Is., Ryukyus, 28. iv. 1958, K. Iha leg.; 5 exs.(paratypes), Takarajima, Is. Tokara, 2. vii. 1960, M. Sato leg.; 1 ex., Takarajima, Tokara Is., Japan, 18. vii. 1964, Y. Hama leg.

3. G onocephalum takara Nakane, 1963

Spec. exam.: 2 exs.(holo-\& paratypes), Takarajima, Tokara Is., 2. vii. 1960, Y. Hama leg. ; 2 exs.(1 paratype), Takarajima, Is. Tokara, 2. vii. 1960, M. Sato leg. ; 1 ex., id., 3. vii. 1960, M. Sato leg. ; 3 exs. 
(paratypes), Is. Takarajima, Tokara Islands, 2. vi. 1962, M. Sato leg. ; 1 ex., Takarajima, Tokara Is., Japan, 16. vii. 1964, A. Tanaka leg. ; 2 exs., id., 17. vii. 1964, H. Shima leg. ; 1 ex., Takarajima-Is., Tokara-Islands, 13. vii. 1964, A. Tanaka leg. ; 3 exs., id., 17. vii. 1964, A. Tanaka leg.; 1 ex., id., 20. vii. 1964, S. Ohga leg.

4. Caedius maderi Kaszab, 1964

Spec. exam. : 6 exs., Takarajima, Is. Tokara, 2. vii. 1960, M. Sato leg. ; 18 exs., id., 3. vii. 1960, M. Sato leg. ; 1 ex., Takarajima, Tokara Is., Japan, 18. vii. 1964, H. Shima leg.

\section{Trachyscelini}

5. Trachyscelis chinensis Champion, 1894

Spec. exam.: None (recorded by Z. Kaszab, 1964)

\section{Phalerini}

†6. Micropedinus algae Lewis, 1894

Spec. exam. : 1 ex., Takarajima, Is. Tokara, 3. vii. 1960, M. Sato leg.

\section{Diaperini}

7.Diaperis sanguinipennis sinensis Gebien, 1925

Spec. exam. : 1 ex., Takarajima, Tokara Is., 2. vii. 1960, Y. Hama leg.

8. Platydema pallidicolle (Lewis, 1894)

Spec. exam. : 2 exs., Takarajima, Is. Tokara, 3. vii. 1960, M. Sato leg.

9. Platydema subfascia subfascia (Walker, 1894)

Spec. exam. : 1 ex., Takarajima, Is. Tokara, 2. vii. 1960, M. Sato leg.

10. Ceropria induta induta (Wiedemann, 1819)

Spec. exam.: 2 exs., Takarajima-Is., Tokara-Islands, 15. vii. 1964, H. Shima leg.

11. Pentaphyllus philippinensis Kaszab, 1956

Spec. exam.: None (recorded by T. Nakane, 1968)

\section{Ulomini}

12. Eutochia lateralis Boheman, 1859

Spec. exam.: None (recorded by Z. Kaszab, 1964). 
Cnodalonini

13. Phaedis (Phaedis) helopioides purpurascens Nakane, 1968

Spec. exam.: 3 exs., Takarajima, Tokara Is., Japan, 22. vi. 1973, H. Makihara leg.

\section{Strongyliini}

14. Strongylium cultellatum Mäklin, 1866

Spec. exam.: 1 ex., Takarajima, Tokara Is., 3. vii. 1960, Y. Hama leg.

KIKAI JIMA I.

\section{Diaperini}

†1. P latydema marseuli Lewis, 1894

Spec. exam. : 1 ex., Kikaijima, Amami Is., Ryukyus, 20. v. 1973, H. Makihara leg.

\section{AMAMI-OSHIMA I.}

\section{Pedinini}

1. Diphyrrhinchus shibatai Kaszab, 1964 Spec. exam.: 2 exs., Asani, Amami Is., 19. v. 1960, T. Shibata leg.

2. M esomorphus villiger (Blanchard, 1853)

Spec. exam. : 1 ex., Shinmura, Amami-Oshima, Japan, 23. vii. 1954, S. Miyamoto \& Y. Hirashima leg.

\section{Opatrini}

3. G onocephalum coriaceum Motschulsky, 1857 Spec. exam.: None (recorded by Z. Kaszab, 1952).

4. Gonocephalum okinawanum M. T. Chûjô, 1963 Spec. exam.: 2 ex s., Akagina, Amami-Oshima, 17. vii. 1954, S. Miyamoto \& Y. Hirashima leg. ; 1 ex., Asani, Amami Is., 19. v. 1960, T. Shibata leg.

\section{Phalerini}

5. M icropedinus pallidipennis Lewis, 1894

Spec. exam.: 3 exs., Asani, Amami Is., 19. v. 1960, T. Shibata leg.; 1 ex., Ikari, Amami Is., 21. v. 1960, T. Shibata leg. 
6. Epiphaleria atriceps Lewis, 1894

Spec. exam.: 7 exs., Asani, Amami Is., 19. v. 1960, T. Shibata leg.

Bolitophagini

†7. Dicraeosis carinatus carinatus Gebien, 1913

Spec. exam. : 1 ex., Ôgachi, Amami, Ryukyu Is., 26. iv. 1968, H. Makihara leg. ; 9 exs., Nishinakama, Amami, Ryukyu, 26-28. vii. 1969, H. Makihara leg. ; 6 exs., id., 3-5. vii. 1969, H. Makihara leg.; 3 exs., Hatsuno, Amami-Oshima, Kagoshima, Japan, 30. vi. 1975, H. Makihara leg. ; 1 ex., Nishinakama; Amami-Oshima, Kagoshima, Japan, 1-4. vii. 1975, H. Makihara leg.

8. Byrsax niponicus Lewis, 1894

Spec. exam.: None (recorded by Z. Kaszab, 1964).

\section{Diaperini}

9. Diaperis lewisi intersecta Gebien, 1913

Spec. exam.: 4 exs.: Naze, Amami-Oshima, 19. vii. 1954, S. Miyamoto \& Y. Hirashima leg.

10. Scaphidema kondoi Nakane, 1968

Spec. exam.: None (recorded by T. Nakane, 1968).

11. Scaphidema pictipenne Lewis, 1894

Spec. exam. : 1 ex., Ikari, Amami Is., 22. v. 1960, T. Shibata leg.; 1 ex., Mt. Takada-yama, Amami-Oshima, Nansei Is., Japan, 15. iv. 1970, H. Irie leg.

12. Platydema fumosum kawaii Nakane, 1968

Spec. exam. : 1 ex., Hatsuno, Amami-Oshima, 25. v. 1960, T. Shibata leg.

nec. $P$. fumosum Lewis ; M. T. Châjô, 1966, Jour. Fac. Agr. Kyushu Univ. 14 (1): 11-12.

13. Platydema marseuli Lewis, 1894

Spec. exam. : 1 ex., Ikari, Amami Is., 4. vi. 1960, T. Shibata leg.; 1 ex., Hatsuno, Amami I., Ryukyus, 19. iv. 1974, H. Makihara leg.

14. Platydema pallidicolle (Lewis, 1894)

Spec. exam.: 11 exs., Yuwan-Shinmura, Amami-Oshima, 22. vii. 1954, S. Miyamoto \& Y. Hirashima leg.

15. Platydema subfascia subfascia (Walker, 1858)

Spec. exam.: 2 exs., Ikari, Amami Is., 11. v. 1960, T. Shibata leg.; 2 exs., id., 17. v. 1960, T. Shibata leg.; 2 exs., id., 18. v. 1960, T. Shibata leg. ; 1 ex., id., 22. v. 1960, T. Shibata leg. ; 1 ex., Nishinakama, Amami-Oshima, Japan, 1-4. vii. 1975, H. Makihara leg. 
16. Basanus amamianus M. T. Chûjô, 1966

Spec. exam.: 6 exs. (holo-\& paratypes), Ikari, Amami-Oshima, 22. v. 1960, T. Shibata leg.

NOTE: From this island $\boldsymbol{Z}$. Kaszab recorded $\boldsymbol{B}$. presuturalis Pic in 1964 based on a specimen collected by Mr. T. Shibata (Ikari, Amami-Oshima, 22. v. 1960). But later I got an opportunity to examine 6 specimens collected at the same time with the one which was examined by $Z$. Kaszab. As a result I concluded that these specimens should be separated from $\boldsymbol{B}$. presuturalis Pic, and I treated them as a new taxon named $\boldsymbol{B}$. amamianus in my previous paper (1966, Jour. Fac. Agr., Kyushu Univ. 14 (1) : 13-14, PI. I \& II.). So I like to omit the record of $\boldsymbol{B}$. presuturalis Pic from this island.

17. Ceropria induta induta (Wiedemann, 1819)

Spec. exam.: 2 exs., Nishinakama, Amami, Ryukyu, 26-28. vii. 1969, H. Makihara leg. ; 1 ex., Hatsuno, Amami I., Ryukyus, 19. iv. 1974, H. Makihara leg.; 1 ex., Nishinakama, Amami-Oshima, Kagoshima, Japan, 1-4. vii. 1975, H. Makihara leg.; 2 exs., Nishinakama, Amami I., Ryukyus, 10. iv. 1976, H. Makihara leg.

18. Addia latior Nakane, 1963

Spec. exam. : 1 ex., Yuwandake, Amami-Oshima, 29. vii. 1963, J. L. Gressitt leg. ; 2 exs., id., 30. vii. 1963, Y. Hirashima leg. ; 1 ex., id., 6. viii. 1963, T. Okada leg.

\section{Leiochrini}

19. Derispia japonica Kaszab, 1961

Spec. exam.: 2 exs., Ikari, Amami I., 3. vii. 1961, K. Yamada leg.; 1 ex., (Mt.)Yuwan-dake, 300-600 m, Amami-Oshima I., 29. vii. 1963, J. L. Gressitt leg.

20. Derispia klapperichi Kaszab, 1942

Spec. exam.: None (recorded by M. Miyatake, 1961).

\section{Ulomini}

21. Gnathocerus cornutus Fabricius, 1798

Spec. exam.: 1 ex., Naze, Amami-Oshima, 12. vii. 1933, T. Esaki \& K. Yasumatsu leg.

22. Tribolium (Tribolium) castaneum (Herbst, 1797)

Spec. exam. : 1 ex., Santaro-pass, Amami-Is., 7. v. 1960, T. Shibata leg.

23. Uloma excisa excisa Gebien, 1913 
Saec. exam.: 1 ex., Ohgachi, Amami, Ryukyu Is., 26. iv. 1968, H. Makihara leg. ; 3 exs., Ashikebu, Amami-Oshima, Nansei Is., Japan, 27. v. 1970, H. Makihara leg. ; 3 exs., id., 9. vi. 1970, H. Makihara leg.; 2 exs., Hatsuno, Amami-Oshima, Nansei Is., Japan, 9. iv. 1972, H. Irie leg.; 3 exs., id., 18. iv. 1974, H. Makihara leg.; 6 exs., id., 19. iv. 1974, H. Makihara leg. ; 1 ex., Yuwan-dake, Amami-Oshima, Kagoshima, Japan, 28-29. vi. 1975, H. Makihara leg.; 2 exs., Nishinakama, Amami-Oshima, Kagoshima, Japan, 1-4. vii. 1975, H. Makihara leg. ; 27 exs., Nishinakama, Amami I., Ryukyus, 10. iv. 1976, H. Makihara leg. ; 4 exs., id., 11. iv. 1976, H. Mikihara leg.

24. Uloma ichoi Nakane, 1956

Spec. exam. : 1 ex., Naze, Amami Is., 5. v. 1960, T. Shibata leg.;

1 ex., Ashikebu, Amami, Ryukyu Is., 23. iv. 1968, H. Makihara leg. $\uparrow_{25}$. Alphitobius diaperinus (Panzer, 1797)

Spec. exam. : 1 ex.(light trap), Nishinakama, Amami I., Ryukyus, 7. vi. 1970, H. Makihara leg.

26. Leptoscapha unifasciata Kaszab, 1964

Spec. exam. : 1 ex.(holotype), Ikari, Amami-Oshima, 22. v. 1960,

T. Shibata leg.; 1 ex.(paratype), id., 30. vi. 1960, T. Shibata leg.

27. Hypophloeus (Paraphloeus) amamiensis Kaszab, 1964 Spec. exam. : 2 exs.(holo- \& paratype), Kominato, Amami Is., 29. v. 1960, K. Yamada leg.

\section{Tenebrionini}

28. Setenis oshimanus Miwa, 1935

Spec. exam. : 3 exs., Nishinakama, Amami, Ryukyu, 1-7. vi. 1970, H. Makihara leg. ; 1 ex., Naze, Amami I., Ryukyus, 27. vi. 1973, H. Makihara leg. ; 1 ex.(light), Naze, Amami-Oshima, Kagoshima, Japan, 3. vii. 1975, H. Makihara leg.

29. Menephilus arciscelis Marseul, 1876

Spec. exam.: 1 ex., Sakibaru, Amami Is., 26. vi. 1960, T. Shibata leg. ; 1 ex., Nishinakama, Amami, Ryukyu, 1-7. vi. 1970, H. Makihara leg.; 2 exs., Hatsuno, Amami I., Ryukyus, 19. iv. 1974, H. Makihara leg. ; 1 ex., Nishinakama, Amami I., Ryukyus, 10. iv. 1976, H. Makihara leg.

†30. Catapiestus subrufescens Pic, 1911

Spec. exam. : 1 ex., Mt. Yuwan-dake 300-600 m, Amami Oshima I., 29. vii. 1963, J. L. Gressitt leg. ; 1 ex., Nishinakama, Amami I., Ryukyus, 10. iv. 1976, H. Makihara leg.

31. Cryphaeus satoi Kaszab, 1964 
Spec. exam. : 1 ex., Nishinakama, Amami I., Ryukyus, 23. v. 1973, H. Makihara leg.

\section{Cnodalonini}

32. Tetraphyllus amamiensis Kaszab, 1964

Spec. exam. : 1 ex., Nase, Amami, Ryukyu Is., 7. viii. 1969, H. Makihara leg.; 3 exs., Naze, Amami-Oshima, Nansei Is., Japan, 26. v. 1970, H. Makihara leg. ; 2 exs., Nishinakama, Amami, Ryukyu, 1-7. vi. 1970, H. Makihara leg.; 2 exs., Hatsuno, Amami-Oshima, Nansei Is., Japan, 3. vii. 1970, H. Irie leg.; 1 ex., id., 9. iv. 1972, H. Irie leg.; 1 ex., Hatsuno, Amami-I., Ryukyus, 12. iv. 1976, H. Makihara leg.

33. Tetraphyllus lunuliger laevis subsp. nov.

These specimens examined here are closely related to T.lunuliger (Marseul, 1876), but may clearly be separated from the latter through the following characters :

\section{T. lunuliger lunuliger}

Upper surface densely, strongly punctured ; propleura strongly rugose ; in metasternum apical triangle area which is separated by metafemoral line strongly rugose ; metepisternum roughly punctured ; parameres of male genitalia gently deflexed.

Length : 5.2-6.5 mm. Width : 3.6$4.5 \mathrm{~mm}$.

Distribution : Honshu, Shikoku, Kyushu, Tanegashima I. \& Yakushima I.

\section{T. lunuliger laevis}

Upper surface sparsely, weakly punctured ; propleura nearly smooth ; in metasternum apical triangle area which is separated by metafemoral line very feebly rugose ; metepisternum very sparsely aciculate; parameres of male genitalia reflexed.

Length : 5.6-6.9 mm. Width : 4.0$5.0 \mathrm{~mm}$.

Distribution : Amami-Oshima I., Tokunoshima I. \& Okinawa I.

Type materials : Holotype ơ (Type No. 2059, Kyushu Univ.), Nishinakama, Amami I., Ryukyus, IO. iv. 1976, H. Makihara leg. Paratopotypes: $0^{7} \&$ ㅇ, 23. v. 1973, H. Makihara leg. ; 오, same data as holotype. Paratypes : 2s or \& + , Inokawa, Tokunoshima., Ryukyu, 28-31. v. 1970, H. Makihara leg.; $5 \sigma^{\urcorner} \&$ \& ㅇ, Oku, Okinawa I., Nansei Is., Japan, 31. v. 1974, H. Irie leg.; ㅇ, Mt. Nago-dake, Okinawa I., Nansei Is., Japan, 5. vi. 1974, H. Irie leg. ; ơ \& 9 , Hiji, Okinawa I., Nansei Is., Japan, 6. iv. 1975, H. Irie leg.

Type depository: Holotype is preserved in the Entomological Laboratory, Faculty of Agriculture, Kyushu University. 
34. Hemicera alternata nodokai Nakane, 1963

Spec. exam. : 1 ex., Ikari, Amami Is., 6. v. 1960, T. Shibata leg.;

3 exs., Ashikebu, Amami, Ryukyu Is., 25. iv. 1968, H. Makihara leg. ;

1 ex., Hatsuno, Amami I., Ryukyus, 17. iv. 1974, H. Makihara leg.;

1 ex., Naze, Amami I., Ryukyus, 20. iv. 1974, H. Makihara leg.; 2 exs., Nishinakama, Amami-Oshima, Kagoshima, Japan, 1-4. vii. 1975, H. Makihara leg.

35. Hemicera f ukiensis Kaszab, 1951

Spec. exam.: 1 ex., Nishinakama, Amami, Ryukyu, 26-28. vii. 1969, H. Makihara leg.; 3 exs., Nishinakama, Amami-Oshima, Kagoshima, Japan, 1-4. vii. 1975, H. Makihara leg.

36. Phaedis (Phaedis) helopioides amamianus subsp. nov.

Z. Kaszab (1964) and I (1966) recorded P. (P.) helopioides Pascoe from this island. But later I reexamined the specimens treated by them, and as a result I came to the conclusion that these specimens should be separated from helopioides. Therefore, the population from AmamiOshima I. is described here as P. $(P$.) helopioides amamianus.

On the other hand, T. Nakane described P. (P.) helopioides purpurascens based on the specimens collected on Takarajima I., AmamiOshima I. and Tokunoshima I. in 1968. The two forms, so far as the specimens from Amami-Oshima I. are concerned, may be separated from each other by the following characters:

\section{$P$. (P.) helopioides purpurascens}

Generally larger than amamianus in size (length: $7.1-10.3 \mathrm{~mm}$. width: 3.0-4.4 mm.) ; punctures on vertex to occiput denser; pronotal margin moderately rounded ; elytra comparatively strongly aciculate ; parameres of male genitalia weakly reflexed from their bases.

Distribution : Takarajima I. \& Tokunoshima I.

\section{$P$.(P.) helopioides amamianus}

Generally smaller than purpurascens in size (length : 6.3-9.6 mm. width : 2.7-4.0 mm.) ; punctures on vertex to occiput sparser; pronotal margin very weakly rounded ; elytra very feebly, sparsely aciculate ; parameres of male genitalia continuously, roundly deflexed from body of genitalia.

Distribution : Amami-Oshima I.

Type materials : Holotype or (Type No. 2060, Kyushu Univ.), Yuwandake, Amami I., Ryukyus, 11. v. 1972, Y. Yoshiyasu leg. Paratypes: or \& 2 우으, Ashikebu, Amami, Ryukyu, 27. v. 1970, H. Mikihara leg.; ơ, Nishinakama, Amami, Ryukyu, 1-7. vi. 1970, H. Makihara leg. ; 2 o $^{7} o^{7}$, Hatsuno, Amami, Ryukyu, 5. vi. 1970, H. Makihara leg. ; $\sigma^{7}$, Nishinakama, 
Amami I., Ryukyus, 22. v. 1973, H. Makihara leg. ; + , Mt. Yuwan, Amami I., Ryukyus, 23. iii. 1974, K. Ueda, M. Takagi \& Y. Yoshiyasu leg.; o \& 우, Yuwan-dake, Amami-Oshima, Kagoshima, Japan, 28-29. vi. 1975, H. Makihara leg. ; 오 Nishinakama, Amami-Oshima, Kagoshima, Japan, 1-4. vii. 1975, H. Makihara leg.; ㅇ, Naze, Amami I., Ryukyus, 13. v. 1976, H. Makihara leg.

Type depository: Holotype is preserved in the Entomological Laboratory, Faculty of Agriculture, Kyushu University.

37. Metaclisa nagaii Nakane, 1968

Spec. exam.: 1 ex., Yuwan, Amami Is., 7. viii. 1961, K. Yamada leg.; 1 ex., Nishinakama, Amami-Oshima, Kagoshima, Japan, 1-4. vii. 1975, H. Makihara leg.

38. Obriomaia palpalis palpaloides Nakane, 1964 Spec. exam.: 2 exs.(at light), Naze, Amami I., Ryukyus, 27. vi. 1973, H. Makihara leg. ; 1 ex., Yuwan-dake, Amami-Oshima, Kagoshima, Japan, 28-29. vi. 1975, H. Makihara leg.

39. Obriomaia rufiventris Kaszab, 1964

Spec. exam. : 1 ex., Taken, Amami, Ryukyu Is., 27. iv. 1968, H. Makihara leg. ; 2 exs., Nishinakama, Amami, Ryukyu, 3-5. viii. 1969, H. Makihara leg. ; 1 ex., Ashikebu, Amami-Oshima, Nansei Is., Japan, 27. v. 1970, H. Makihara leg.; 2 exs., Nishinakama, Amami, Ryukyu, 1-7. vi. 1970, H. Makihara leg.; 2 exs., Hatsuno, Amami-Oshima, Nansei Is., Japan, 9. iv. 1972, H. Irie leg. ; 1 ex., Nishinakama, Amami I., Ryukyus, 22. v. 1973, H. Makihara leg. ; 1 ex., id., 23. v. 1973, H. Makihara leg.; 1 ex., Hatsuno, Amami I., Ryukyus, 18. iv. 1974, H. Makihara leg.; 1 ex., Naze, Amami I., Ryukyus, 20. iv. 1974, H. Makihara leg.; 1 ex., Hatsuno, Amami-Oshima, Kagoshima, Japan, 30. vi. 1975, H. Makihara leg. ; 1 ex., Nishinakama, Amami, Ryukyu, 11. iv. 1976, H. Makihara leg. ; 1 ex., Hatsuno, Amami I., Ryukyus, 12. iv. 1976, H. Makihara leg.

40. Obriomaia semiviolacea Nakane, 1968

Spec. exam. : 1 ex., Hatsuno, Amami-Oshima, Nansei Is., Japan, 15. v. 1972, H. Irie leg.

41. Pseudonautes purpurivittatus (Marseul, 1876)

Spec. exam.: 15 exs., Nishinakama, Amami, Ryukyu, 26-28. vii. 1969, H. Makihara leg.; 4 exs., id., 3-5. viii. 1969, H. Makihara leg. ; 1 ex., Mt. Yuwan, Is. Amami-Oshima, 25. vi. 1971, Coll. M. Chûjô ; 2 exs., Nishinakama, Amami I., Ryukyus, 22. v. 1973, H. Makihara leg. ; 3 exs., Yuwan-dake, Amami-Oshima, Kagoshima, Japan, 28-29. vi. 1975, H. Makihara leg. ; 6 exs., Hatsuno, Amami-Oshima, Kagoshima, 
Japan, 30. vi. 1975, H. Makihara leg.; 5 exs., Nishinakama, AmamiOshima, Kagoshima, Japan, 1-4. vii. 1975, H. Makihara leg.

\section{Adeliini}

42. Laena rotundicollis insularis Kaszab, 1964

Spec. exam.: 1 ex., Nishinakama, Amami, 16. vi. 1970, H. Makihara leg.; 1 ex.(holotype), Ikari, Amami Is., 18. v. 1960, T. Shibata leg.

\section{Helopini}

43. Tarpela amamiensis Kaszab, 1964

Spec. exam.: 1 ex., Kominato, Is. Amami-Oshima, Ryukyus, 24. iii. 1968, K. Ioki leg.; 13 exs., Hatsuno, Amami, Ryukyu Is., 29. iv. 1968, H. Makihara leg. ; 7 exs., Nishinakama, Amami, Ryukyus, 1-7. vi. 1970, H. Makihara leg. ; 1 ex.(light trap), Naze, Amami I., Ryukyus, 25. v. 1973, H. Makihara leg.; 2 exs., Hatsuno, Amami I., Ryukyus, 17. iv. 1974, H. Makihara leg.; 1 ex., id. 19. iv. 1974, H. Makihara leg.; 8 exs., Nishinakama, Amami, Ryukyu, Il. iv. 1976, H. Makihara leg.; 1 ex., Hatsuno, Amami I., Ryukyus, 12. iv. 1976, H. Makihara leg.

\section{Amarygmini}

\section{Plesiophthalmus brevipennis Lewis, 1896}

Spec. exam.: 2 exs., Ashikebu, Amami-Oshima, Nansei Is., Japan, 27. v. 1970, H. Makihara leg. ; 2 exs., Nishinakama, Amami, Ryukyu, 1-7. vi. 1970, H. Makihara leg.; 1 ex., Hatsuno, Amami-Oshima, Kagoshima, Japan, 30. vi. 1975, H. Makihara leg. ; 2 exs., Nishinakama, Amami-Oshima, Kagoshima, Japan, 1-4. vii. 1975, H. Makihara leg.

45. Plesiophthalmus mizusawai Yamazaki, 1968

Spec. exam.: None (recorded by H. Yamazaki, 1968).

NOTE: I recorded Plesiophthalmus fuscoaenescens Fairmaire from this island (1966, Jour. Fac. Agr., Kyushu Univ. 14 (1) :27-28.), but this was my wrong arrangement in manuscription.

46. Elixota iridicollis Nakane, 1968

Spec. exam.: 1 ex.(at light), Naze, Amami I., Ryukyus, 27. vi. 1973, H. Makihara leg. ; 1 ex.(at light), Amami, Ryukyus, 26. vi. 1974, K. Setoya \& K. Ueda leg.; 2 exs., Nishinakama, Amami-Oshima, Kagoshima, Japan, 1-4. vii. 1975, H. Makihara leg.

\section{Strongyliini}

47. Strongylium amamianum Nomura, 1963 
Spec. exam.: 1 ex., Ikari, Amami Is., 16. vi. 1961, T. Shibata leg.; 1 ex., id., 21. vi. 1961, T. Shibata leg. ; 1 ex., id., 30. vi. 1961, T. Shibata leg. ; 1 ex., Hatsuno, Amami Is., 11. vii. 1961, T. Shibata leg.; 1 ex., Yuwan-dake, Amami-Oshima, Kagoshima, Japan, 28-29. vi. 1975, H. Makihara leg. ; 1 ex., Hatsuno, Amami-Oshima, Kagoshima, Japan, 30. vi. 1975, H. Makihara leg.; 7 exs., Nishinakama, AmamiOshima, Japan, 1-4. vii. 1975, H. Makihara leg.

48. Strongylium cultellatum Mäklin, 1866

Spec. exam. : 2 exs., Yuwan-dake 550 m, Amami-Oshima, 18. vii. 1963, C. M. Yoshimoto leg.

49. Strongylium marseuli yuwanum Nomura, 1963

Spec. exam.: 2 exs., Ikari, Amami Is., 2. vii. 1961, T. Shibata leg.; 1 ex., id., 3. vii. 1961, T. Shibata leg. ; 1 ex., id., 4. vii. 1961, T. Shibata leg. ; 4 exs., Hatsuno, Amami-Oshima, Nansei Is., Japan, 3. vii. 1970, H. Irie leg.; 8 exs., Nishinakama, Amami-Oshima, Nansei Is., Japan, 1-4. vii. 1975, H. Makihara leg.

NOTE: In male of this species eyes extremely closed each other and comparatively, widely separated from each other in female.

50. Strongylium oshimanum Fairmaire, 1897

Spec. exam. : 1 ex., (Mt.)Yuwan-dake 550 m, Amami-Oshima I., 17. vii. 1963, C. M. Yoshimoto leg. ; 24 exs., Nishinakama, Amami-Oshima, Kagoshima, Japan, 1-4. vii. 1975, H. Makihara leg.

51. Strongylium shibatai Nomura, 1963

Spec. exam.: None (recorded by S. Nomura, 1963).

52. Ainu masumotoi Nakane, 1963

Spec. exam.: 1 ex., Shinmura-Yuwan, Amami-Oshima, 4. iv. 1956, S. Miyamoto leg.

53. E ucrossoscelis broscosomoides Nakane, 1963

Spec. exam.: 1 ex.(paratype), Santaro-pass, Amami Is., 30. v. 1960, T. Shibata leg.; 11 exs., Hatsuno, Amami-Oshima, Nansei Is., Japan, 9. iv. 1972, H. Irie leg. ; 2 exs., id., 14. v. 1972, H. Irie leg.

TOKUNOSHIMA I.

\section{Phaleriini}

\section{${ }^{\dagger}$. M icropedinus pallidipennis Lewis, 1894}

Spec. exam.: 1 ex., Mennawa, Tokunoshima I., 30. vii. 1968, K. Nozato leg.

Diaperini

†2. Platydema subfascia subfascia (Walker, 1858) 
Spec. exam.: 20 exs., Inokawa, Tokunoshima, Ryukyu, 28-31. v. 1970, H. Makihara leg.

+3. Ceropria induta induta (Wiedemann, 1819)

Spec. exam.: 4 exs., Inokawa, Tokunoshima, Ryukyu, 28-31. v. 1970, H. Makihara leg.

\section{Ulomini}

4. Uloma excisa excisa Gebien, 1913

Spec. exam.: 3 exs., Mikyo, Is. Tokuno-shima, Ryukyus, 11. iv. 1968, K. Ioki leg. ; 34 exs., Inokawa, Tokunoshima, Ryukyu, 28-31. v. 1970, H. Makihara leg.

\section{Tenebrionini}

5. Menephilus arciscelis Marseul, 1876 Spec. exam. : 16 exs., Inokawa, Tokunoshima, Ryukyu, 28-31. v. 1970, H. Makihara leg.

6. Setenis oshimanus Miwa, 1935

Spec. exam. : 1 ex., Mikyo 200 m, Tokunoshima, 27. vii. 1963, J. L. Gressitt leg.

\section{Cnodalonini}

†7. Tetraphyllus lunuliger laevis M. T. Chûjô Spec. exam.: Mentioned above (3 paratypes).

†. Hemicera alternata nodokai Nakane, 1963 Spec. exam.: 3 exs., Inokawa, Tokunoshima, Ryukyu, 28-31. v. 1970, H. Makihara leg.

†9. Hemicera f ukiensis Kaszab, 1951

Spec. exam. : 1 ex., Inokawa, Tokunoshima, Ryukyu, 28-31. v. 1970, H. Makihara leg.

IO. Phaedis (Phaedis) helopioides purpurascens Nakane, 1968 Spec. exam.: None (recorded by T. Nakane, 1969).

11. Pseudonautes purpurivittatus (Marseul, 1876) Spec. exam.: 6 exs., Inokawa, Tokunoshima, Ryukyu, 28-31. v. 1970, H. Makihara leg.

\section{Amarygmini}

12. Plesiophthalmus makiharai sp. nov.

Oblong oval, dark brown with bluish tinge, tarsi paler. Head wholly covered with yellow pubescence, strongly, densely punctured ; clypeus gently, roundly deflexed forwards ; widely depressed along clypeal suture ; 
preocular area strongly reflexed, weakly expanded outwards ; interocular area with shallow, longitudinal groove. Eye very large, feebly convex. Pronotum roundly convex, base widest, gently narrowed forwards, base : front $=$ ca. $3: 2$, sparsely aciculate, wholly covered with golden pubescence; front margin narrowly emarginated, lateral margin weakly, roundly expanded, emarginated like front margin, base not emarginated. Scutellum nearly triangular, very sparsely aciculate. Elytra strongly convex, narrowly emarginated, widest at $3 / 5$ from base, punctate-striae very feebly grooved, interstices very sparsely, weakly aciculate.

Antennae reaching to hind coxae; 1st very thick, strongly thickened towards apex, 2nd very short, 3rd longest, relative length of each segment (base to apex)=6 $: 2: 19: 10: 15: 12: 11: 9: 8: 8: 9$. Prosternum sparsely punctured, with comparatively long pubescence ; mesosternum with dense punctures and pubescence ; metasternum nearly smooth, with sparse pubescence. Abdominal sternite wholly covered with long, dense pubescence ; apical margin of last visible abdominal sternite widely, deeply sinuated in male.

Profemur weakly flattened, thickest at $2 / 3$ from base, with obtuse femoral projection, and covered with sparse, short pubescence; mesoand metafemur sparsely punctured, evenly pubescent. Protibia weakly

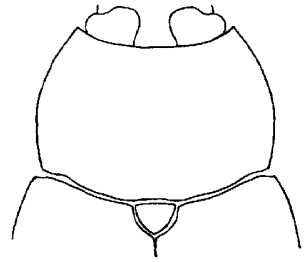

I a

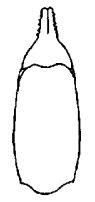

$\mathrm{I} b$

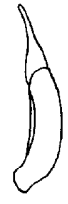

$1 \mathrm{c}$

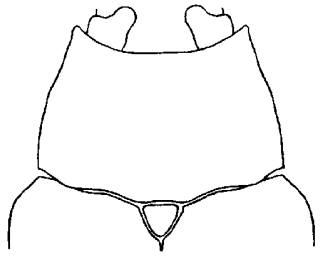

2 a

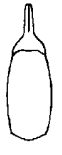

$2 b$

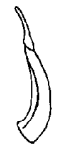

$2 \mathrm{c}$

Fig. 1. Plesiophthalmus makiharai sp. nov. la: Dorsal view of pronotum. lb: Dorsal view of male genitalia. 1c: Lateral view of male genitalia.

Fig. 2. P. brevipennis Lewis. 2a: Dorsal view of pronotum. 2b: Dorsal view of male genitalia. 2c: Lateral view of male genitalia. 
dilated towards apex, very weakly warped inwards, apical half of inside with dense, brushy hairs; mesotibia very weakly warped ; metatibia feebly waved. Every tarsus with dense, brushy hairs on sole, even on apical segment. Relative length of each protarsal segment (base to apex $=22: 6: 4: 14$. Male genitalia very thick, apical $2 / 3$ of parameres strongly narrowed towards apex, side of narrowed part with many hook-shaped, thick and short spines bending towards base.

Length : 12.0-13.0 mm. Width : 6.2-6.6 mm.

Distribution : Tokunoshima Island.

Type materials: Holotype or (Type No. 2061, Kyushu Univ.), Inokawa, Tokunoshima, Ryukyu, 28-31. v. 1970, H. Makihara leg. Paratopotypes $\sigma^{\pi} \&$ 웅, same data as holotype.

Type depository: Holotype is preserved in the Entomological Laboratory, Faculty of Agriculture, Kyushu University.

This species is very close to $P$. brevipennis Lewis, 1896 from AmamiOshima I., but they are separated from each other through the following characters :

P. brevipennis Lewis

Interocular area nearly flat ; front corner of pronotum angulated, aciculations on pronotum and elytra extremely sparser; apical part of last visible abdominal sternite very feebly depressed ; male genitalia much smaller, parameres much narrower, hook-shaped spines on parameres much smaller.

\section{P. makiharai sp. nov.}

Interocular area with longitudinal groove ; front corner of pronotum not so angulated, aciculations on pronotum and elytra comparatively denser; apical part of last visible abdominal sternite widely, deeply sinuated ; male genitalia much larger, parameres much wider, hookshaped spines on parameres much larger.

OKINOERABU JIMA I.

\section{Bolitophagini}

\section{$\dagger 1$ D icraeosis carinatus carinatus Gebien, 1913}

Spec. exam. : 8 exs., Okinoerabu I., Ryukyu Is., 31. vii. - 2. viii. 1969, H. Makihara leg. ; 4 exs., Ohyama, Okinoerabu, Nansei Is., Japan, 12. vii. 1970, H. Irie leg.; 5 exs., Oyama, Okinoerabu I., 3. vii. 1974, H. Makihara leg. ; 1 ex., id., 4. vii. 1974, H. Makihara leg.; 3 exs., id., 6. vii. 1974, H. Makihara leg. 
Diaperini

†2. Platydema subfascia subfascia (Walker, 1858) Spec. exam.: 2 exs., Ohyama, Okinoerabu, Nansei Is., Japan, 19. v. 1972, H. Irie leg.

\section{Ulomini}

†3. Uloma bonzica bonzica Marseul, 1876

Spec. exam. : 1 ex., Okinoerabu I., Ryukyu Is., 31. vii. - 2. viii. 1969, H. Makihara leg.

†4. Alphitobius diaperinus (Panzer, 1797)

Spec. exam. : 2 exs., Okinoerabu I., Ryukyu Is., 31. vii. - 2. viii. 1969, H. Makihara leg.

\section{Cnodalonini}

†5. O briomaia palpalis palpaloides Nakane, 1963

Spec. exam.: 2 exs., Ohyama, Okinoerabu, Nansei Is., Japan, 19. v. 1972, H. Irie leg. ; 1 ex., id., 20. v. 1972, H. Irie leg. ; 1 ex., Wadomari, Okinoerabu I., Ryukyus, 2. vii. 1974, H. Makihara leg.; 1 ex., Oyama, Okinoerabu I., Ryukyus, 6. vii. 1974, H. Makihara leg.

\section{Strongyliini}

†6. Strongylium amamianus Nomura, 1963

Spec. exam. : 1 ex., Okinoerabu I., Ryukyu Is., 31. vii. - 2. viii. 1969, H. Makihara leg. ; 1 ex., Ohyama, Okinoerabu, Nansei Is., Japan, 12. vii. 1970, H. Irie leg.

\section{Acknowledgements}

I wish to express my appreciation to Prof. Y. Hirashima, the head of Hikosan Biological Laboratory, for making this study possible and for his kind advice in the preparation of the manuscript. Also, I express my cordial thanks to Prof. Emeritus K. Yasumatsu, Prof. T. Shirôzu and Assoc. Prof. K. Yano of Kyushu University for their continual kind guidance. Paticular thanks are due to Prof. Emeritus M. Chûjô of Kagawa University for his various help to my study. My hearty thanks are due to Mr. H. Makihara of Kyushu University and Mr. H. Irie of Fukuoka City who kindly gave me so many valuable Ryukyuan tenebrionid specimens. 